\title{
International Standards for Neurological and Functional Classification of Spinal Cord Injury
}

Frederick M Maynard, Jr, Michael B Bracken, Graham Creasey, John F Ditunno, Jr, William H Donovan, Thomas B Ducker, Susan L Garber, Ralph J Marino, Samuel L Stover, Charles H Tator, Robert L Waters, Jack E Wilberger and Wise Young

American Spinal Injury Association, 2020 Peachtree Road, NW Atlanta Georgia 30309, USA

The first edition of the International Standards for Neurological and Functional Classification of Spinal Cord Injury, ie neural disturbances ('Spinal Cord Injury') whether from trauma or disease, was published in $1982^{6}$ by the American Spinal Injury Association (ASIA). Reference was made to the 1992 Revision of the International Standards and published in Paraplegia (the former title of Spinal Cord) in 1994, Volume 32, pages $70-80$ by JF Ditunno Jr, W Young, WH Donovan and G Creasey ${ }^{7}$. Since then there have been three revisions, the most recent being in 1996. Dr Frederick M Maynard Jr in his Preface to this Fourth Revision said:-

Since the earlier revision of the "ASIA Standards' was completed in 1992, it has received endorsement by the International Medical Society of Paraplegia (IMSOP) and has been subsequently known as the International Standards for Neurological and Functional Classification of Spinal Cord Injury (ISCSCI-92). As a result of the enthusiastic response from the international medical rehabilitation community to these standards, there has been close scrutiny of their every word and concept. The changes in the fourth revision are the 1992 Committee's response to feedback from the wider community of spinal cord injury clinicians and researchers for greater precision in definition and clarification of points of ambiguity.

Since 1994, a Training Package of four video tapes and a 143 page Reference Manual have been completed by members of the 1992 committee. This package is available from the ASIA Publications office and is strongly recommended to all clinicians and researchers who have a serious commitment to record neurological impairments in a standardized manner. The Reference Manual is both a learning guide for new users of the Standards and an authoritative reference text for experienced users. The committee also hopes that its content will promote investigations that produce empirical data on which to base further revisions.

In consideration of the anticipated need for further revisions based on new information, the
ASIA Board has established a standing committee to reevaluate regularly the need for further modifications in the Standards booklet and in the Training Package, as well as to respond to questions and criticisms of the Standards from the many users. This committee welcomes correspondence that raises questions, offers constructive criticism or provides new empirical data that is relevant for further refinements and improvements in the reliability and validity of the ISCSCI.

Frederick M Maynard, Jr MD Chairperson

ASIA Neurological Standards Committee August 1996

Permission has been very kindly given by Dr James Keene, President of the American Spinal Injury Association for publication of the 1996 Revision in Spinal Cord. Copies of the booklet and training package can be obtained by contacting: Lesley $\mathrm{M}$ Hudson MA, 2020 Peachtree Road NW, Atlanta, Georgia 30309, USA.

\section{Introduction}

The spinal cord is the major conduit through which motor and sensory information travels between brain and body. The spinal cord contains longitudinally oriented spinal tracts (white matter) surrounding central areas (gray matter) where most spinal neuronal cell bodies are located. The gray matter is organized into segments comprising sensory and motor neurons. Axons from spinal sensory neurons enter and axons from motor neurons leave the spinal cord via segmental nerves or roots. The roots are numbered and named according to the foramina through which they enter/exit the vertebral column. For example, the two C6 roots (left and right) pass through foramina situated between the $\mathrm{C} 5$ and $\mathrm{C} 6$ vertebrae.

Each root receives sensory information from skin areas called dermatomes. Similarly, each root innervates a group of muscles called a myotome. While a dermatome usually represents a discrete and contiguous skin area, most roots innervate more than one muscle, and most muscles are innervated by more than one root. 
Spinal cord injury (SCI) affects conduction of sensory and motor signals across the site(s) of lesion(s). By systematically examining the dermatomes and myotomes, as described within this booklet, one can determine the cord segments affected by spinal cord injury. From such an examination, several measures of neurological damage are generated, e.g., Neurological Level, Sensory Level and Motor Level (on right and left sides), Sensory Scores (Pin Prick and Light Touch), Motor Score and Zone of Partial Preservation.

Here we endorse the ASIA Impairment Scale (replacing the modified Frankel Classification of earlier versions of this booklet) and recommend the Functional Independence Measure (the FIM - an approach for assessing the impact of spinal cord injury on the individual's daily life activities and functions). Thus, the FIM data describing disability complement the more traditional neurological and impairment measures.

We begin with basic definitions of common terms used herein. The section that follows describes the recommended neurological examination, both sensory and motor components. The following sections cover sensory and motor scores, the ASIA Impairment Classification, clinical syndromes associated with spinal cord injury, and the FIM. Finally, for ease of reference, a summary chart of the recommended system is included (Figure 5). A fullsize version for photocopying and use in the patient's record may be obtained by contacting: Lesley M Hudson MA, 2020 Peachtree Road, NW Atlanta, Georgia 30309, USA.

\section{Definitions}

Tetraplegia (preferred to'quadriplegia')

This term refers to impairment or loss of motor and/or sensory function in the cervical segments of the spinal cord due to damage of neural elements within the spinal canal. Tetraplegia results in impairment of function in the arms as well as in the trunk, legs and pelvic organs. It does not include brachial plexus lesions or injury to peripheral nerves outside the neural canal.

\section{Paraplegia}

This term refers to impairment or loss of motor and/or sensory function in the thoracic, lumbar or sacral (but not cervical) segments of the spinal cord, secondary to damage of neural elements within the spinal canal. With paraplegia, arm functioning is spared, but, depending on the level of injury, the trunk, legs and pelvic organs may be involved. The term is used in referring to cauda equina and conus medullaris injuries, but not to lumbosacral plexus lesions or injury to peripheral nerves outside the neural canal.
Quadriparesis and paraparesis

Use of these terms is discouraged, as they describe incomplete lesions imprecisely. Instead, the ASIA Impairment Scale (vide intra) provides a more precise approach.

\section{Dermatome}

This term refers to the area of the skin innervated by the sensory axons within each segmental nerve (root).

\section{Myotome}

This term refers to the collection of muscle fibers innervated by the motor axons within each segmental nerve (root).

\section{Neurological level, sensory level and motor level (see summary chart, Figure 4)}

The first of these terms refers to the most caudal segment of the spinal cord with normal sensory and motor function on both sides of the body. In fact, the segments at which normal function is found often differ by side of body and in terms of sensory vs. motor testing. Thus, up to four different segments may be identified in determining the neurological level, ie, R-sensory, Lsensory, R-motor, L-motor. In cases such as this, it is strongly recommended that each of these segments be separately recorded and that a single 'level' not be used, as this can be misleading in such cases. When the term Sensory Level is used, it refers to the most caudal segment of the spinal cord with normal sensory function on both sides of the body; the Motor Level is similarly defined with respect to motor function. These 'levels' are determined by neurological examination of: (1) a key sensory point within each of 28 dermatomes on the right and 28 dermatomes on the left side of the body, and (2) a key muscle within each of 10 myotomes on the right and 10 myotomes on the left side of the body.

\section{Skeletal level}

This term refers to the level at which, by radiographic examination, the greatest vertebral damage is found.

Sensory scores and motor scores (see summary chart, Figure 4)

Numerical summary scores that reflect the degree of neurological impairment associated with the SCI.

\section{Incomplete injury}

If partial preservation of sensory and/or motor functions is found below the neurological level and includes the lowest sacral segment, the injury is defined as incomplete. Sacral sensation includes sensation at the anal mucocutaneous junction as well as deep anal sensation. The test of motor function is the presence of 
voluntary contraction of the external anal sphincter upon digital examination.

\section{Complete injury}

This term is used when there is an absence of sensory and motor function in the lowest sacral segment. ${ }^{15}$

\section{Zone of partial preservation ( $Z P P$ )}

This term refers to those dermatomes and myotomes caudal to the neurological level that remain partially innervated. When some impaired sensory and/or motor function is found below the lowest normal segment, the exact number of segments so affected should be recorded for both sides as the ZPP. The term is used only with complete injuries.

\section{Neurological examination}

\section{Introduction}

The neurological examination has two components (sensory and motor), which are separately described below. Further, the neurological examination has both required as well as optional, though recommended, elements. The required elements are used in determining the sensory/motor/neurological levels, in generating scores to characterize sensory/motor functioning and in determining completeness of the injury. The optional measures, though not used in scoring, may add to a specific patient's clinical description.

\section{When the patient is not fully testable}

When a key sensory point or key muscle is not testable for any reason, the examiner should record 'NT' instead of a numeric score. In such cases, sensory and motor scores for the affected side of the body, as well as total sensory and motor scores, cannot be generated with respect to the injury at that point in treatment. Further, when associated injuries, e.g., traumatic brain injury, brachial plexus injury, limb fracture, etc, interfere with completion of the nuerological examination, the neurological level should still be determined as accurately as possible. However, obtaining the sensory/motor scores and impairment grades should be deferred to later examinations.

\section{Sensory examination: required elements}

The required portion of the sensory examination is completed through the testing of a key point in each of the 28 dermatomes on the right and on the left sides of the body. ${ }^{2}$

At each of these key points, two aspects of sensation are examined: sensitivity to pin prick and to light touch. ${ }^{3}$ Appreciation of pin prick and of light touch at each of the key points is separately scored on a three-point scale:

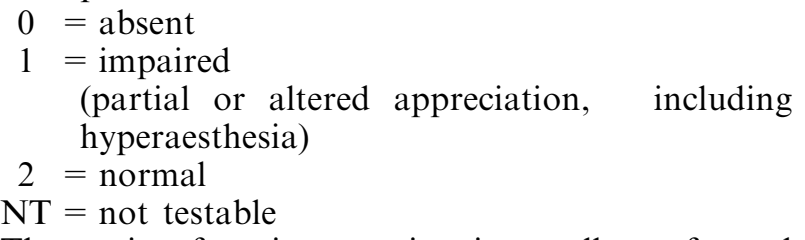

The testing for pin sensation is usually performed with a disposable safety pin; light touch is tested with cotton. In testing for pin appreciation, the inability to distinguish between dull and sharp sensation is graded as 0 .

The following key points are to be tested bilaterally for sensitivity (see Figures 1 and 4). Asterisks indicate that the point is at the mid-clavicular line:

C2-Occipital protuberance

C3-Supraclavicular fossa

C4-Top of the acromioclavicular joint

C5-Lateral side of the antecubital fossa

C6-Thumb

C7-Middle finger

C8-Little finger

T1-Medial (ulnar) side of the antecubital fossa

T2-Apex of the axilla

T3-Third intercostal space (IS)*

T4-Fourth IS (nipple line)*

T5-Fifth IS (midway between T4 and T6)*

T6-Sixth IS (level of xiphisternum)*

T7-Seventh IS (midway between T6 and T8)*

T8-Eighth IS (midway between T6 and T10)*

T9-Ninth IS (midway between T8 and T10)*

T10-Tenth IS (umbilicus)*

T11-Eleventh IS (Midway between T10 and T12)*

T12-Inguinal ligament at mid-point

L1-Half the distance between T12 and L2

L2-Mid-anterior thigh

L3-Medial femoral condyle

L4-Medial malleolus

L5-Dorsum of the foot at the third metatarsal phalangeal joint

S1-Lateral heel

S2-Popliteal fossa in the mid-line

S3-Ischial tuberosity

S4-5 Perianal area (taken as one level)

In addition to bilateral testing of these key points, the external anal sphincter should be tested through insertion of the examiner's finger; perceived sensation should be graded as being present or absent (ie, enter Yes or No on the patient's summary chart). This information is needed in determining completeness/ incompleteness of injury.

\section{Sensory examination: optional elements}

For purposes of SCI evaluation, the following aspects of sensory function are defined as optional (though they are strongly recommended): position sense and awareness of deep pressure/deep pain. If these are examined, it is recommended that they be 


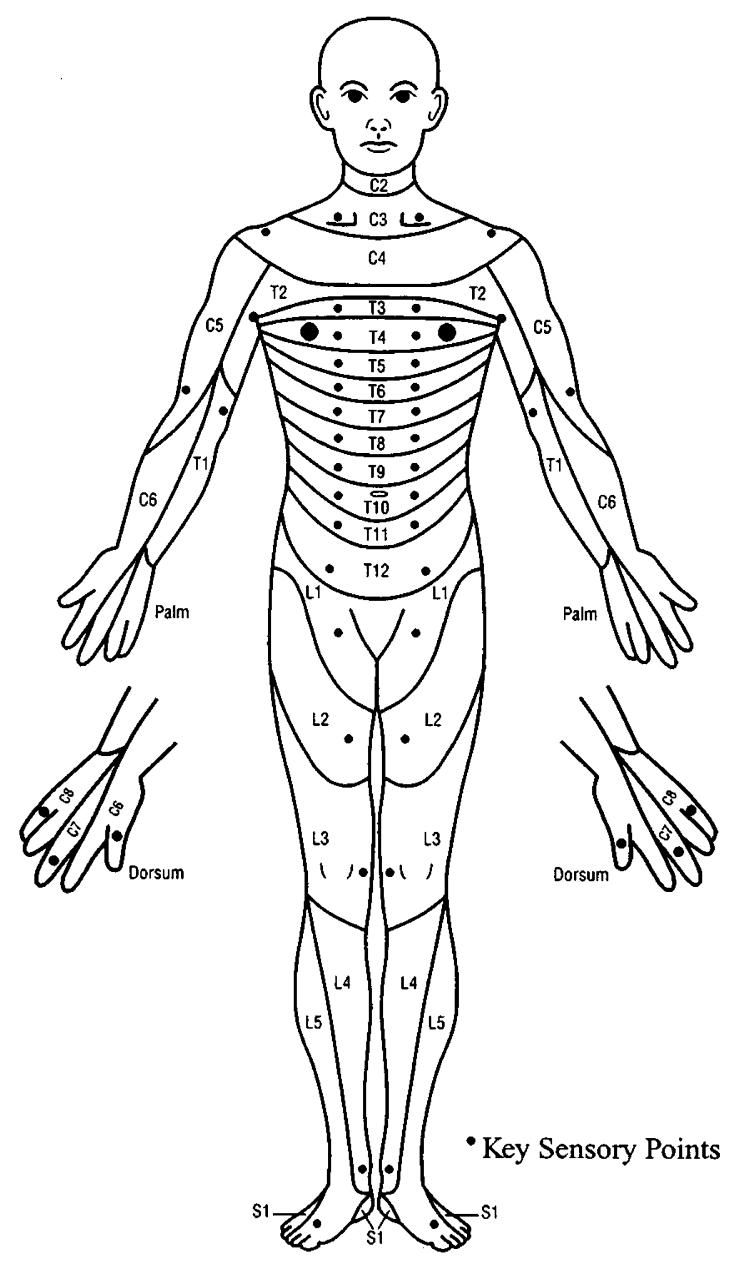

Figure 1

graded using the sensory scale provided herein (absent, impaired, normal). It is also suggested that only one joint be tested for each extremity; the index finger and the great toe of the right and left sides are recommended.

\section{Motor examination: required elements}

The required portion of the motor examination is completed through the testing of a key muscle (one on the right and one of the left side of the body) in the 10 paired myotomes (see below). Each key muscle should be examined in a rostral-caudal sequence.

The strength of each muscle is graded on a six-point scale: $:^{1,4,5,11}$

$0=$ total paralysis

$1=$ palpable or visible contraction

$2=$ active movement, full range of motion (ROM) with gravity eliminated

3 = active movement, full ROM against gravity
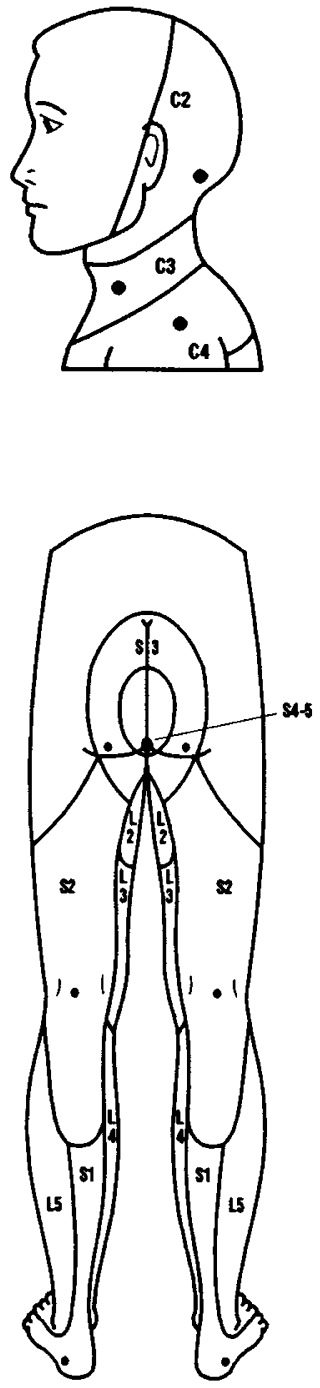

$4=$ active movement, full ROM against moderate resistance

$5=$ (normal) active movement, full ROM against full resistance

$\mathrm{NT}=$ not testable

The following muscles are to be examined (bilaterally) and graded using the scale defined above. The muscles were chosen because of their consistency for being innervated by the segments indicated and their ease of testing in the clinical situation, where testing in any position other than the supine position may be contraindicated.

C5-Elbow flexors (biceps, brachialis)

C6-Wrist extensors (extensor carpi radialis longus and brevis)

C7 - Elbow extensors (triceps)

$\mathrm{C} 8$ - Finger flexors (flexor digitorum profundus) to the middle finger

T1 - Small finger abductors (abductor digiti minimi)

L2 - Hip flexors (iliopsoas) 
L3 - Knee extensors (quadriceps)

L4-Ankle dorsiflexors (tibialis anterior)

L5 - Long toe extensors (extensor hallucis longus)

S1-Ankle plantarflexors (gastrocnemius, soleus)

For those myotomes that are not clinically testable by a manual muscle exam, i.e., $\mathrm{C} 1$ to $\mathrm{C} 4, \mathrm{~T} 2$ to $\mathrm{L} 1$ and $\mathrm{S} 2$ to S5, the motor level is presumed to be the same as the sensory level.

In addition to bilateral testing of these muscles, the external anal sphincter should be tested on the basis of contractions around the examiner's finger and graded as being present or absent (i.e., enter Yes or No on the patient's summary sheet). This latter information is used solely for determining the completeness of injury.

\section{Motor examination: optional elements}

For purposes of SCI evaluation, it is recommended that other muscles be evaluated, but their grades are not used in determining the motor score or motor level. It is particularly suggested that the following muscles be tested: (1) diaphragm (via fluoroscopy), (2) deltoids, (3) abdominals (via Beevor's Sign), (4) media hamstrings, (5) hip adductors. Their strength is to be described as absent, weak or normal.

\section{Sensory and motor scores/levels}

Sensory scores and sensory level

Required testing generates four sensory modalities per dermatome: R-pin prick, R-light touch, L-pin prick, Llight touch. As is indicated on the summary chart enclosed, these scores are then summed across dermatomes and sides of body to generate two summary sensory scores: Pin Prick and Light Touch Score. The sensory scores provide a means of numerically documenting changes in sensory function.

Further, through the required sensory examination the sensory components for determining neurological level (i.e. the sensory level), zone of partial preservation and impairment grade are obtained.

Motor scores and motor level ${ }^{12}$

The required motor testing generates two motor grades per paired myotome: Right and Left. As indicated in Figure 4, these scores are then summed across myotomes and sides of body to generate a single summary motor score. The motor score provides a means of numerically documenting changes in motor function.

Further, through the required motor examination, the motor components for determining neurological level (ie, the motor level), zone of partial preservation and impairment grade are obtained.

Motor level determination: further considerations Just as each segmental nerve (root) innervates more than one muscle, most muscles are innervated by more than one nerve segment (usually two segments; see Figure 2). Therefore, the assigning of one muscle or one muscle group (ie, the key muscle) to represent a single spinal nerve segment is a simplification, used with the understanding that in any muscle the presence of innervation by one segment and the absence of innervation by the other segment will result in a weakened muscle.

By convention, if a muscle has at least a grade of 3, it is considered to have intact innervation by the more rostral of the innervating segments. In determining the motor level, the next most rostral key muscle must test as 5, since it is assumed that the muscle will have both of its two innervating segments intact. For example, if no activity is found in the C7 key muscle and the C6 muscle is graded as 3, then the motor level for the tested side of the body is C6, providing the C5 muscle is graded 5 .

The examiner's judgement is relied upon to determine whether a muscle that tests as less than normal (5) may in fact be fully innervated. This may occur when full effort from the patient is inhibited by factors such as pain, positioning and hypertonicity or when weakness is judged to be due to disuse. If any of these or other factors impede standardized muscle testing the muscle should be graded as not testable

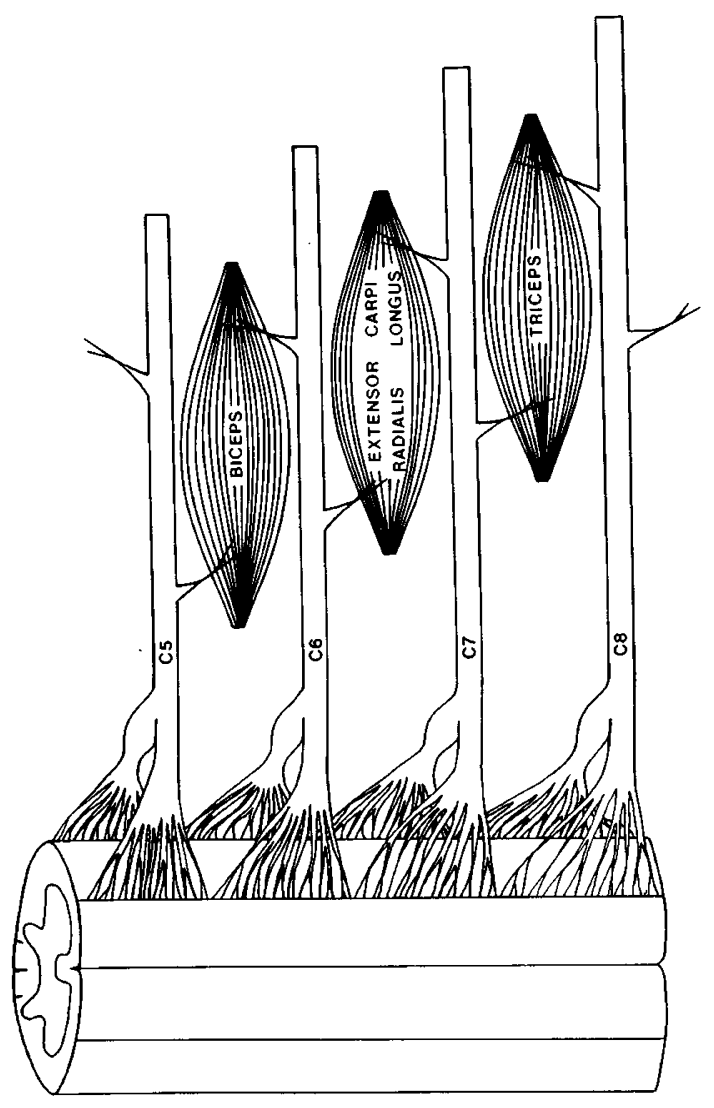

Figure 2 Schematic depiction of innervation of each of three key muscles by two nerve segments 
(NT). However, if these factors do not prevent the patient from performing a forceful contraction and the examiner's best judgement is that the muscle would test normally (5) were it not for these factors, it may be graded as 5 .

In summary, the motor level (the lowest normal motor segment - which may differ by side of body) is defined by the lowest key muscle that has a grade of at least 3, providing the key muscles represented by segments above that level are judged to be normal (5).

\section{Asia impairment scale (modified from Frankel) ${ }^{8,14,15}$}

The following scale is used in grading the degree of impairment:

$A=$ Complete. No sensory or motor function is preserved in the sacral segments S4-S5

$B=$ Incomplete. Sensory but not motor function is preserved below the neurological level and includes the sacral segments S4-S5.

$C=$ Incomplete. Motor function is preserved below the neurological level, and more than half of key muscles below the neurological level have a muscle grade less than 3 .

$D=$ Incomplete. Motor function is preserved below the neurological level, and at least half of key muscles below the neurological level have a muscle grade greater than or equal to 3 .

$E=$ Normal. Sensory and motor function is normal.

\section{Clinical syndromes}

Central cord syndrome

A lesion, occurring almost exclusively in the cervical region, that produces sacral sensory sparing and greater weakness in the upper limbs than in the lower limbs.

\section{Brown-Sequard syndrome}

A lesion that produces relatively greater ipsilateral proprioceptive and motor loss and contralateral loss of sensitivity to pain and temperature.

\section{Anterior cord syndrome}

A lesion that produces variable loss of motor function and of sensitivity to pain and temperature, while preserving proprioception.

Conus medullaris syndrome

Injury of the sacral cord (conus) and lumbar nerve roots within the spinal canal, which usually results in

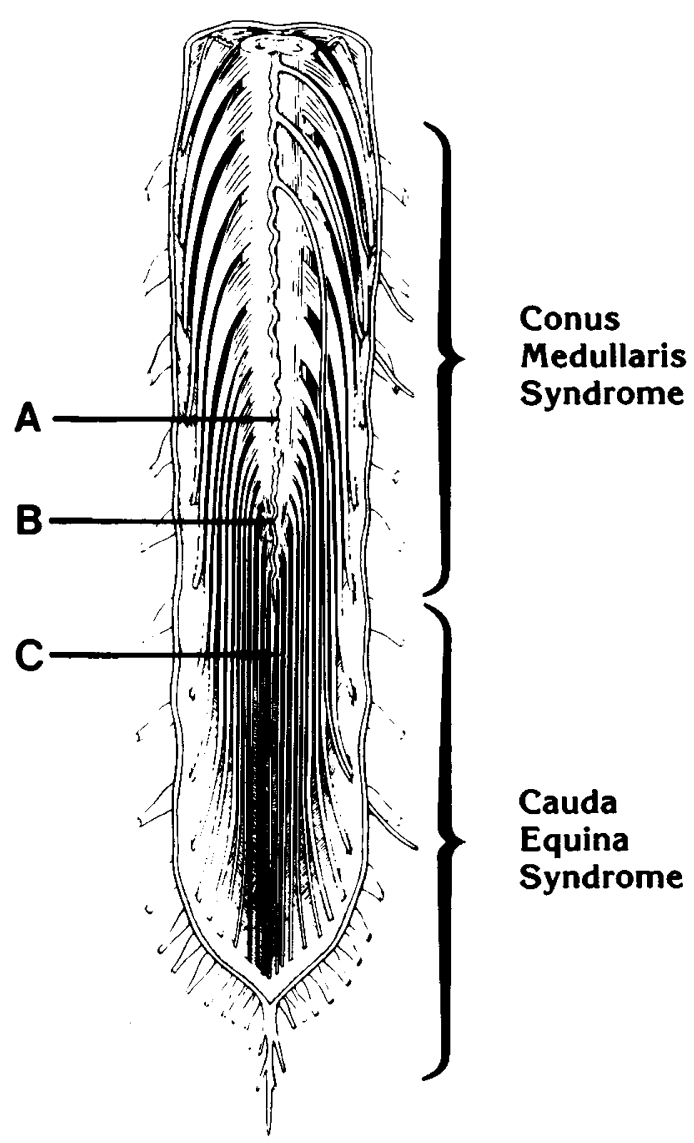

Figure 3

an areflexic bladder, bowel and lower limbs, with lesions as at B in Figure 3. Sacral segments may occasionally show preserved reflexes, eg. bulbocavernosus and micturition reflexes, with lesions as at $\mathrm{A}$ in Figure 3.

Cauda equina syndrome

Injury to the lubosacral nerve roots within the neural canal resulting in areflexic bladder, bowel and lower limbs, with lesions as at $\mathrm{C}$ in Figure 3.

\section{Functional independence measure (FIM)}

To fully describe the impact of SCI on the individual and to monitor/evaluate progress associated with treatment, a standard measure of daily-life activities is necessary. The Functional Independence Measure $(\mathrm{FIM})^{9}$ is one approach to functional assessment that has become widely utilized in the U.S. and is gaining acceptance internationally.

The FIM focuses on six areas of functioning: selfcare, sphincter control, mobility, locomotion, communication and social cognition. Within each area, two or more specific activites/items are evaluated, with a total of 18 items. For example, six activity items (eating, 
grooming, bathing, dressing-upper body, dressinglower body, and toileting) comprise the self-care area (see Figure 4).

Each of the 18 items is evaluated in terms of independence of functioning, using a seven-point scale:

Independent (no human assistance is required):

$7=$ Complete independence: The activity is typically performed safely, without modification, assistive devices or aids, and within reasonable time.

$6=$ Modified independence: The activity requires an assistive device and/or more than reasonable time and/ or is not performed safely.

Dependent (human supervision or physical assistance is required):

$5=$ Supervision or setup: No physical assistance is needed, but cuing, coaxing or setup is required.

$4=$ Minimal contact assistance: Subject requires no more than touching and expends $75 \%$ or more of the effort required in the activity.

$3=$ Moderate assistance: Subject requires more than touching and expends $50-75 \%$ of the effort required in the activity.

$2=$ Maximal assistance: Subject expends $25-50 \%$ of the effort required in the activity.
$1=$ Total assistance: Subject expends $0-25 \%$ of the effort required in the activity.

Thus, the FIM total score (summed across all items) estimates the cost of disability in terms of safety issues and of dependence on others and on technological devices. The profile of area scores and item scores pinpoints the specific aspects of daily living that have been most affected by SCI.

In using the FIM with individuals who have experienced SCI, it should be kept in mind that the FIM was developed for the disabled population in general. It samples those areas of activity that have been found to be affected by impairment among diverse disability groups. Although basic issues of reliability and validity of the FIM have been explored by the developers, ${ }^{10}$ its validity as an instrument for precisely gauging changed functioning with all SCI subpopulations has yet to be demonstrated empirically. For example, it is not yet clear that the self-care items sensitively gauge changes in self-care functioning experienced by tetraplegics during the course of rehabilitation. Further, the reliability estimates for the communication and social cognition areas have been found to be lower than for other areas assessed. Despite these caveats, the use of the FIM is recommended, as it is relatively simple to use, reflects

STANDARD NEUROLOGICAL CLASSIFICATION OF SPINAL CORD INJURY
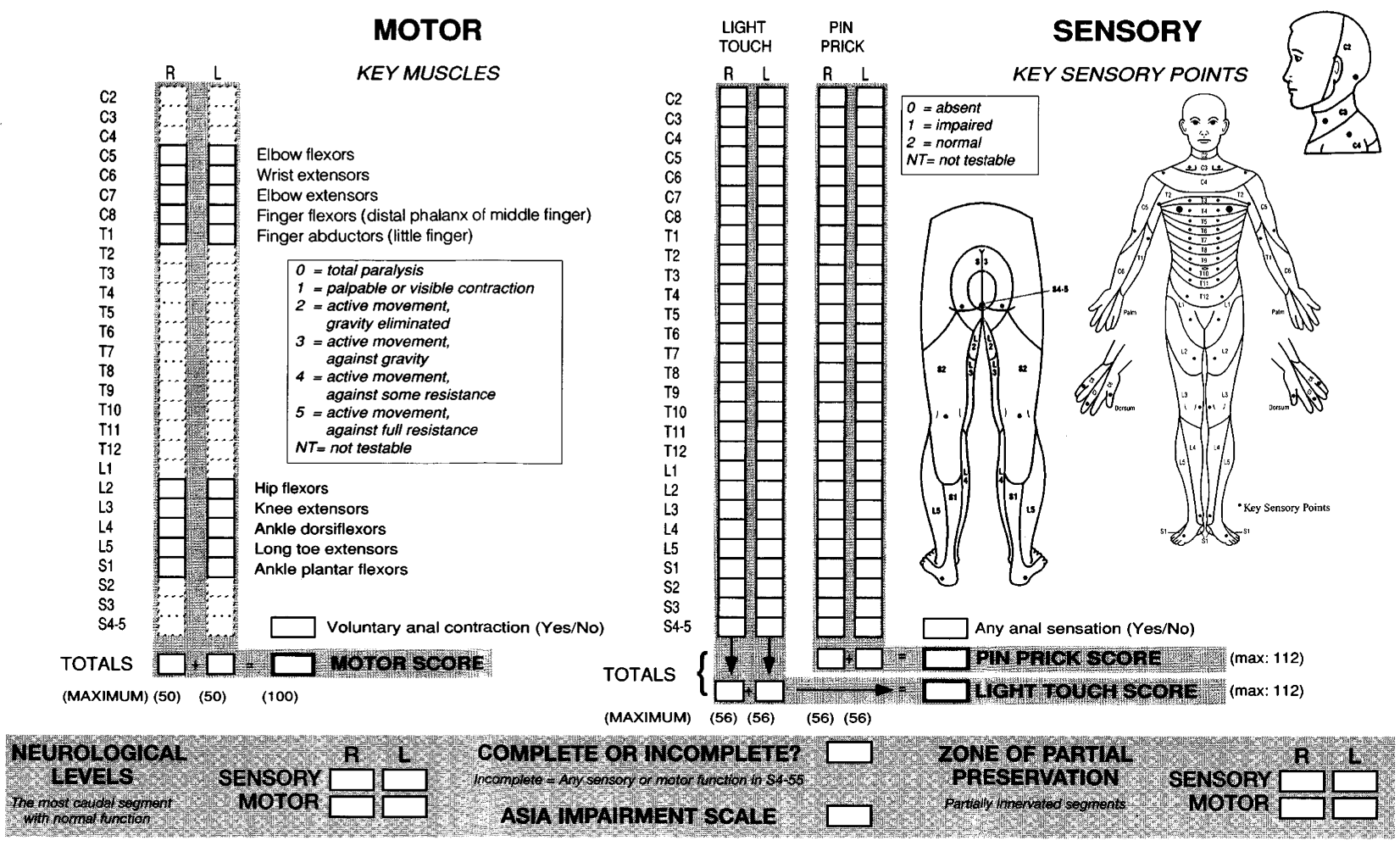

This form may be copied freely but should not be altered without permission from the American Spinal Injury Association. 
Functional Independence Measure (FIM)

\begin{tabular}{|l|l|l|}
\hline & 7 Complete Independence (Timely, Safely) & $\begin{array}{l}\text { No } \\
\text { Helper }\end{array}$ \\
\hline L Modified Independence (Device) & Modified Dependence \\
\hline E & 5 Supervision \\
E & Minimal Assist (Subject $=75 \%+$ ) \\
L & Moderate Assist (Subject $=50 \%+$ ) \\
S Complete Dependence & Helper \\
2 Maximal Assist (Subject $=25 \%+$ ) \\
1 Total Assist (Subject $=0 \%+$ )
\end{tabular}

Figure 5

functional issues of importance to SCI, and guidelines for its use have been carefully developed. ${ }^{6}$

Specific instructions for use of the FIM can be obtained directly from the developers of the FIM. Request the 'Guide for Use of the Uniform Data Set for Medical Rehabilitation (1990),' at the following address: Uniform Data System for Medical Rehabilitation, State University of New York, 232 Parker Hall, 3435 Main Street, Buffalo, New York 14214-3009 USA

The copyright of the International Standards Booklet and of this article on it in Spinal Cord by ASIA/IMSOP must be noted:

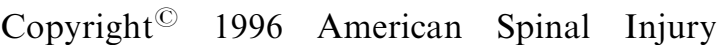
Association (ASIA) and Uniform Data System for Medical Rehabilitation $\left(\mathrm{UDSMR}^{\mathrm{SM}}\right.$ ) a division of UB Foundation Activities, Inc. The booklet and this article on standards are intended for the express use of training professionals in the use of the International Standards for Neurological and Functional Classification of Spinal Cord Injury. No part of the publications may be modified, reproduced, stored in a retrieval system, or transmitted in

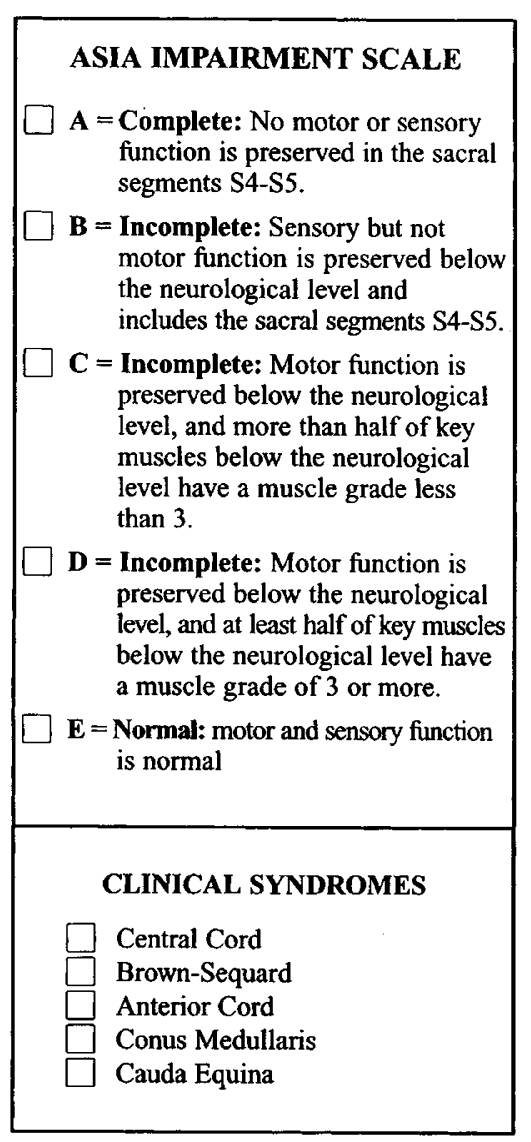

any form or by any means, electronic, photocopying, recording or otherwise, without prior written permission of both ASIA and UDSMR. All rights reserved.

The only portion of this booklet to which this prohibition of modification, reproduction, storage in a retrieval system or transmission in any form does not apply is the front page of the tri-fold pullout sheet, called the 'Standard Neurological Classification of Spinal Cord Injury' worksheet. Notice is found at the bottom of that page which attests to the permission granted by ASIA for duplication, but alteration of this form in any manner is prohibited without permission from ASIA.

Editors note (Mr Phillip Harris): It will be noted that in pages 275 to 281 of this issue of Spinal Cord there is further reference to these International Standards. It is interesting and important that since their first publication increasing use is being made by doctors of these International Standards for research studies and scientific articles on spinal cord disorders, non-traumatic and also traumatic. 


\section{References}

1 Aids to Investigation of Peripheral Nerve Injuries. Medical Research Council War Memorandum, 2nd ed., Revised. London, HMSO, 1943.

2 Austin GM.: The Spinal Cord: Basic Aspects and Surgical Considerations. 2nd ed., p. 762. Springfield, IL: Thomas, 1972.

3 Bracken MB, Shepard MJ, Collins WF, Holford TR et al.: A randomized controlled trial of methylprednisolone or naloxone in the treatment of acute spinal cord injury. $N$ Engl J Med 1990; 332: $1405-11$.

4 Brunnstrom F, Dennen M.: Round table on muscle testing. Annual Conference of American Physical Therapy Association Federation of Crippled and Disabled, Inc. New York 1931:1-12.

5 Daniels L, Worthingham C.: Muscle Testing: Techniques of Manual Examination. 3rd ed. Philadelphia: Saunders, 1972.

6 Ditunno JF.: Functional assessment in CNS trauma. J Neurotrauma 1992; 9: S301-S305.

7 Ditunno JF, Young W, Donovan WH, Creasey G. The International Standards Booklet for Neurological and Functional Classification of Spinal Cord Injury. Paraplegia 1994; 32: $70-80$.

8 Frankel HL, Hancock DO, Hyslop G et al:: The value of postural reduction in the initial management of closed injuries of the spine with paraplegia and tetraplegia. Paraplegia 1969; 7(3): 179-92.

9 Hamilton BB, Fuhrer MJ. (eds) In: Rehabilitation Outcomes: Analysis and Measurement. Baltimore: Brooks, 1987:137-47.

10 Hamilton BB, Laughlin JA, Granger CV, Kayton RM. Interrater agreement of the seven level Functional Independence Measure (FIM). Arch Phys Med Rehabil 1991; 72: 790.

11 Lovett RW.: The Treatment of Infantile Paralysis. 2nd ed., p. 136 Philadelphia: P. Blakiston's Son, 1917.

12 Lucas JT, Ducker TB.: Motor classification of spinal cord injuries with mobility, morbidity and recovery indices. Am Surg 1979; 45: $151-8$.

13 Michaelis LS.: International inquiry on neurological terminology and prognosis in paraplegia and tetraplegia. Paraplegia 1969; 7: $1-5$.

14 Tator CH, Rowed DW, Schwartz ML. (eds): Sunnybrook cord injury scales for assessing neurological injury and neurological recovery in early management of actue spinal cord injury. New York: Raven Press, 1982:7.

15 Waters RL, Adkins RH, Yakura JS.: Definition of complete spinal cord injury. Paraplegia 1991; 9: 573-81. 